\title{
Bending in extrusion of optical fibre preforms
}

\author{
Y. M. Stokes ${ }^{1}$
}

(Received 14 December 2009; revised 14 April 2010)

\begin{abstract}
An optical fibre is made by stretching, or 'drawing', a glass or polymer preform in a drawing tower. Extrusion through dies has received much recent attention as a suitable method for manufacture of preforms of complex shape and novel material composition. However, the preforms are subject to bending during extrusion, so that the final preform has a bent end that must be removed to yield a straight preform to be drawn into a fibre. Control of bending is needed to improve the efficiency of preform extrusion and reduce wastage of high-cost material. Bending occurs even for simple axisymmetric die geometries and in the absence of heating. Hence, we here examine asymmetry in the applied pressure as a cause of bending. Assuming a linear stress profile, and using a quasi-static approach, we determine the relationship between the magnitude of asymmetry and the amount of bend of the preform, for preforms with circular or annular cross section. We show that significant bend may be caused by an asymmetry that is small relative to the applied pressure. A simple idea for controlling bending is also suggested. Undergraduate university level mathematics is used in the analysis, making this a useful educational illustration of the role of mathematics in understanding engineering problems.
\end{abstract}

http://anziamj . austms.org.au/ojs/index.php/ANZIAMJ/article/view/2334 gives this article, (c) Austral. Mathematical Soc. 2010. Published April 23, 2010. ISSN 1446-8735. (Print two pages per sheet of paper.) 


\section{Contents}

1 Introduction

C125

2 Quasi-static model

C128

3 Analysis of bending

C132

4 Conclusions

C134

References

C135

\section{Introduction}

An optical fibre is made by stretching, or 'drawing', a glass or polymer preform in a drawing tower. A typical preform has a length of $10-30 \mathrm{~cm}$ and a diameter of $1-3 \mathrm{~cm}$ and is intended to be a macroscopic version of the final fibre which will be several kilometres in length and have a diameter of around $100 \mu \mathrm{m}$. A high quality preform is a necessary prerequisite to a high quality fibre, fit for its intended purpose. Automated and consistently reproducible manufacture of high quality preforms is needed for commercial production of microstructured optical fibres having patterns of holes with diameters measured in micrometres in the cross section, and extrusion through metal dies is seen as a promising method for manufacture of the preforms [2].

The experimental extruder used at the Centre of Expertise in Photonics at The University of Adelaide (CEP-UA) for research into preform extrusion is constructed so that the longitudinal axis of the die is vertical and preforms are extruded vertically downwards. However, the preforms are subject to bending. Initially, the preform bends as it exits the die, but as the extruded length increases it straightens, so that the final preform has a bent end. Figure 1 shows an example of this for a simple polymer preform of circular cross section. The bent end must be cut off to yield a straight rod-like preform 


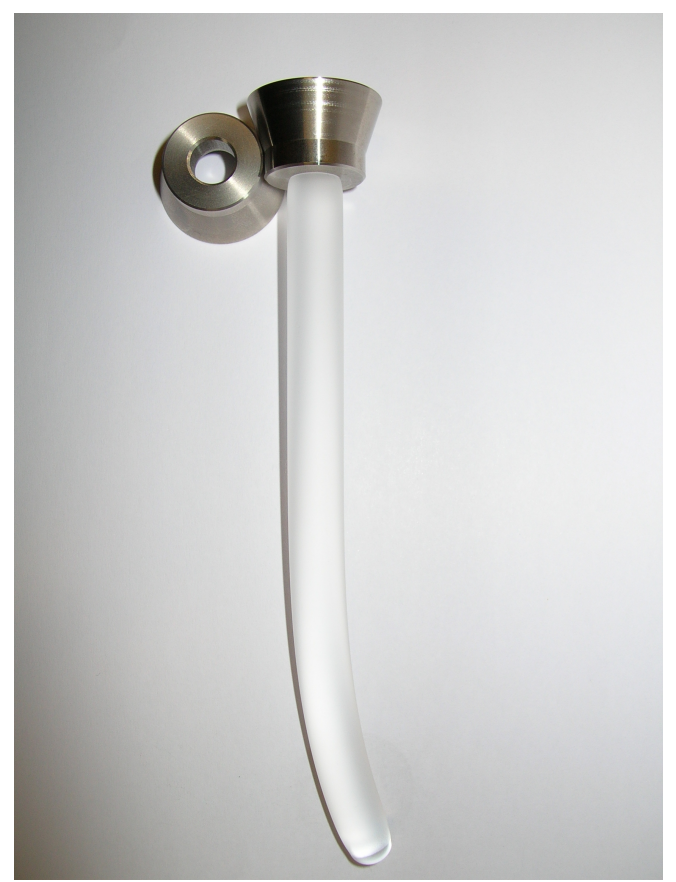

FiguRE 1: An extruded polymer preform of circular cross section (that is, a rod preform). The end furthest from the die, corresponding to the part first extruded, is bent. (Photograph courtesy of the Centre of Expertise in Photonics, The University of Adelaide.)

for drawing into a fibre. In turn, the length of fibre that can be drawn from the preform is reduced; in some instances the preform is completely unusable. This is not only a cause of inefficiency in the production of optical fibre, but there is wastage of high-cost material also.

Bending is seen in other extrusion processes also and understanding the causes and mechanisms for control have received some attention, with nonisothermal temperature and asymmetry in die geometry identified as likely causes $[1,4]$. In extrusion of optical fibre preforms asymmetries in die geometry or temperature profile are potential causes of bending [5], but bending 

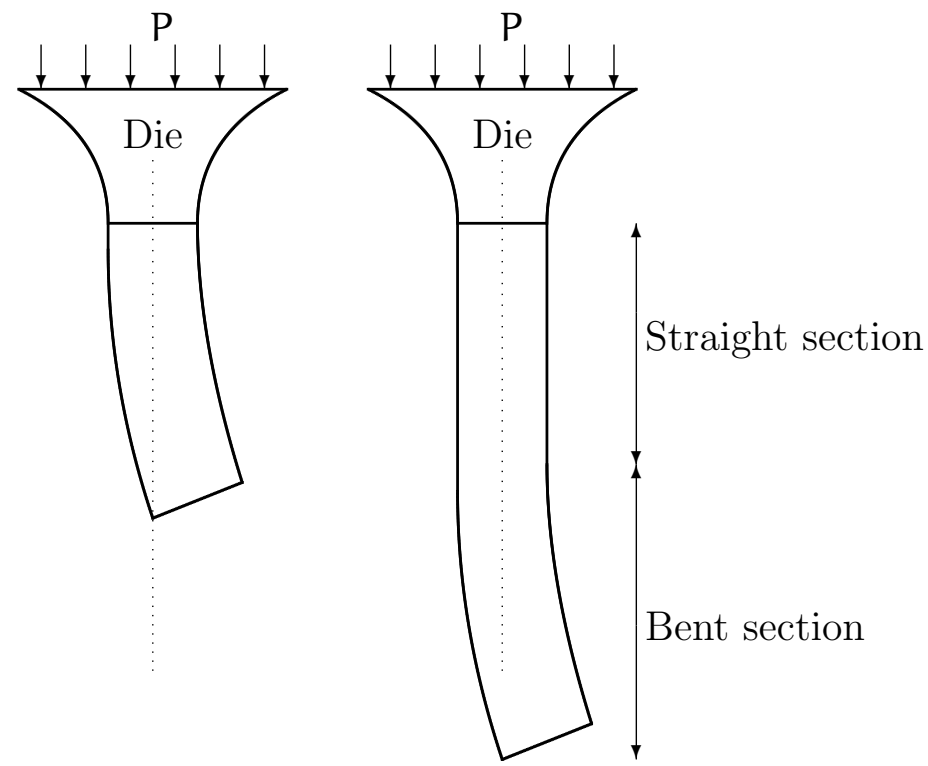

(a) Early time. (b) Later time.

Figure 2: Bending of preform during extrusion.

occurs for simple axisymmetric die geometries and when extruding plasticine in the absence of heating. Thus, noting that high pressures (approximately 10-20 MPa for glass and approximately $1-10 \mathrm{MPa}$ for polymer [3]) are required for preform extrusion, we here investigate asymmetry in the applied pressure as a cause of bending of optical fibre preforms. For this we consider the simplest cases of extruding circular rod or tube preforms and assume an axisymmetric die (although not practically feasible for a preform of annular cross section). We also assume a uniform temperature in any cross section, so that the temperature only varies along the preform, which precludes bending induced by temperature variation.

The mathematics used in our analysis of this problem is at undergraduate university level. The mathematical detail given is intended to facilitate the use of this problem as an educational illustration of the role of mathematics in understanding engineering problems. 


\section{Quasi-static model}

Preforms, especially glass preforms, are extruded very slowly over a period of hours. Most of the applied force is resisted by the die and the extrudate becomes, effectively, solid after exiting the die. This suggests the following explanation for bending.

The stress at the die outlet is, essentially, due to the weight of material hanging below. However, a small asymmetry in the setup of the extruder will lead to a small asymmetry in the stress profile across the die exit to be resolved into a uniform stress plus a moment. The moment causes the preform to move off-centre, that is, to bend, so as to balance the forces and moments. As the weight of the preform below the die exit increases, the amount by which the preform must be off-centre decreases, so that the preform straightens out as its length increases. This is depicted in Figure 2. We examine this explanation as a quasi-static problem. This approach is valid only once the bend has formed but still yields some useful information. In particular, we consider the relationship between the magnitude of asymmetry in the stress distribution and the amount of bend in the preform.

If the setup is axisymmetric, there is a uniform tensile stress $\sigma$ across the die outlet (of area $A$ ), and hence a net force $W=\sigma A$ acting upwards along the axis of the die and preform to balance the weight $W$ of the preform hanging below (Figure 3a). If the stress distribution is non-uniform, the force $W$ acts through a line a distance $d$ from the die axis, which equates to a force $W$ acting along the axis and a moment (or couple) $M=W d$ acting about the axis (Figure 3b).

Consider an annular die of inner radius $a$ and outer radius $b>a$; the special case $a=0$ yields a rod die. Suppose that the stress increases linearly across the die outlet in the direction of the $x$ axis, the origin of which is at the axis of the die, as in Figure 3(b). (The setup of the extruder at the CEP-UA makes this a distinct possibility.) Then the tensile stress distribution is

$$
\sigma(x)=k x+\sigma_{0},
$$



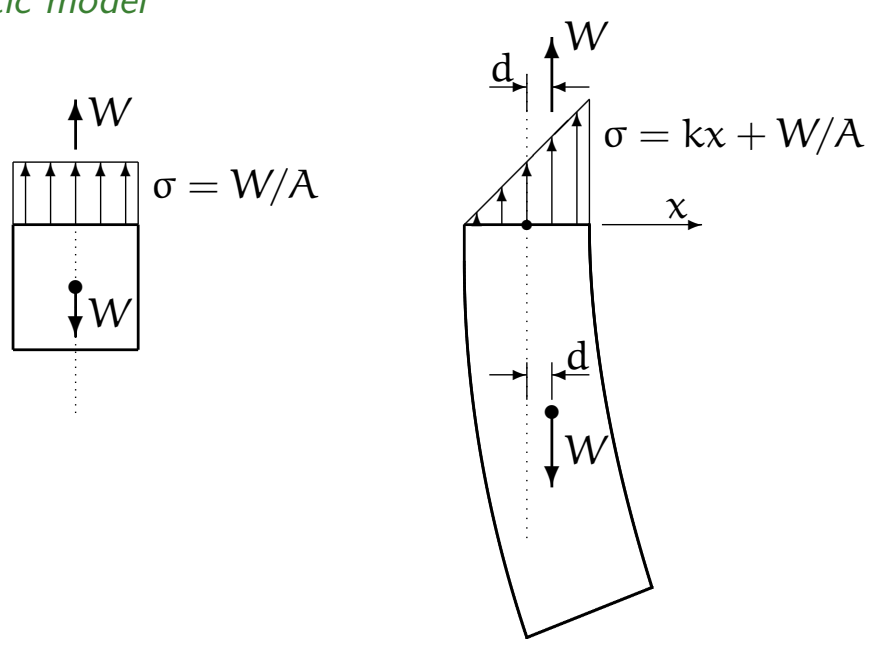

(a) Axisymmetric stress (b) Non-axisymmetric distribution at die outlet. stress distribution at die outlet.

Figure 3: Forces and moments.

where $\sigma_{0}=\sigma(0)=W / A, A=\pi\left(b^{2}-a^{2}\right)$, is the average stress. To determine the moment about $x=0$ consider a circular die outlet of radius $b$ (that is, let $a=0$ ). Next we take a small portion of the outlet area of small width $d x$, spanning the outlet in a direction perpendicular to the $x$ axis, as shown in Figure 4. If this region is located at position $x$, it has area $A=2 \sqrt{b^{2}-x^{2}} d x$. The force acting over this region is $F=\sigma(x) A$ and this yields a moment $F x$ about $x=0$. Integrating (summing) over all such segments from $x=-a$ to $x=a$ yields the total moment resulting from a linear stress profile over a circular die exit

$$
M=\int_{-b}^{b} F x d x=\int_{-b}^{b} 2\left(k x+\sigma_{0}\right) \sqrt{b^{2}-x^{2}} x d x .
$$

For an annular die exit we must subtract the contribution from the circular region of radius $\mathrm{a}$, so that

$$
M=\int_{-b}^{b} 2\left(k x+\sigma_{0}\right) \sqrt{b^{2}-x^{2}} x d x-\int_{-a}^{a} 2\left(k x+\sigma_{0}\right) \sqrt{a^{2}-x^{2}} x d x
$$




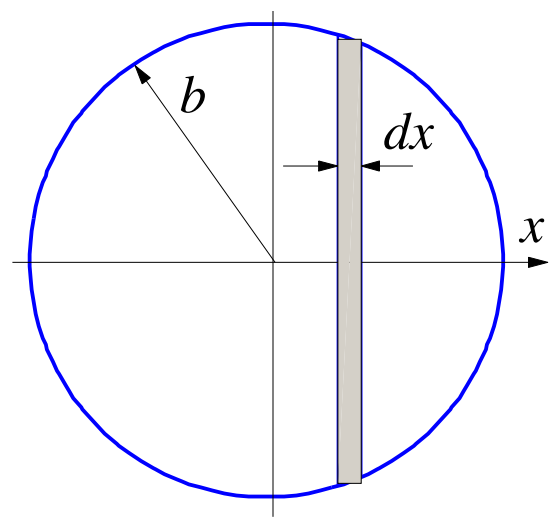

Figure 4: Portion of die outlet area of small width $\mathrm{d} x$ at some position $x$, spanning the outlet in a direction perpendicular to the $x$ axis (shown shaded).

$$
\begin{aligned}
= & 2 k \int_{-b}^{b} x^{2} \sqrt{b^{2}-x^{2}} d x+2 \sigma_{0} \int_{-b}^{b} x \sqrt{b^{2}-x^{2}} d x \\
& -2 k \int_{-a}^{a} x^{2} \sqrt{a^{2}-x^{2}} d x-2 \sigma_{0} \int_{-a}^{a} x \sqrt{a^{2}-x^{2}} d x .
\end{aligned}
$$

The second and fourth terms, due to the average stress $\sigma_{0}$, contribute nothing to the moment, as can be verified by integration or by noting that the integrand is an odd function. The first and third terms are integrated using the substitutions $x=b \sin \theta$ and $x=a \sin \theta$, respectively. The expression obtained must be equal to the moment $\mathrm{Wd}$, yielding

$$
M=W d=\frac{k\left(b^{4}-a^{4}\right) \pi}{4} \quad \text { or } \quad d=\frac{k\left(b^{4}-a^{4}\right) \pi}{4 W} .
$$

As already commented $d$ is the distance along the $x$ axis from the die axis to the line through which the upward force opposing the preform weight is acting. For balance of forces and moments, it must also be the distance from the die centreline to the centre of mass of the preform. Assuming that $k$ remains constant, it is clear that $d$ decreases as the weight $W$ of the preform increases. 


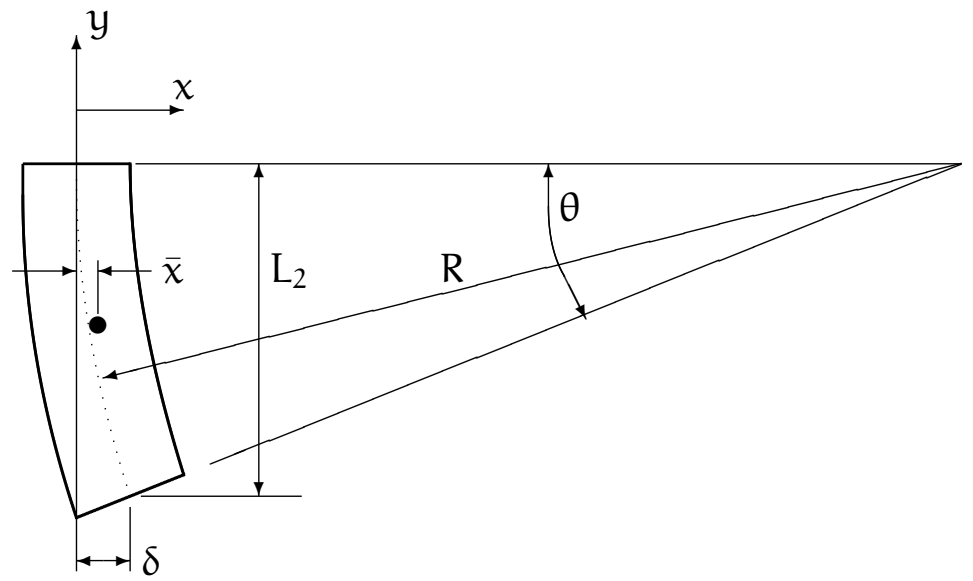

Figure 5: Bend geometry.

We relate the distance $d$ to the shape of the preform as follows. Firstly, we divide the preform hanging below the die outlet into an upper straight section of weight $W_{1}$ and a lower bent section of weight $W_{2}$. Since the axis of the straight section is coincident with the axis of the die, it contributes nothing to the restoring moment $-\mathrm{Wd}$ (in the clockwise direction) balancing the anticlockwise moment $\mathrm{Wd}$ due to the asymmetric stress distribution. Hence, the restoring moment

$$
M=-W d=-\left(W_{1}+W_{2}\right) d=-W_{2} \bar{x},
$$

where $\bar{x}$ is the offset of the centre of mass of the bent section of the preform from the axis of the die. If the bent portion of the preform has length $L_{2}$ and deviation $\delta$ from vertical at the bottom (see Figure 5), and we assume the bend to be a circular arc of radius $\mathrm{R}$ subtending angle $\theta$,

$$
\mathrm{L}_{2}=\mathrm{R} \sin \theta, \quad \delta=\mathrm{R}(1-\cos \theta) .
$$

Then, the offset $\bar{x}$ from the die axis of the centre of mass of the bent portion of the preform is determined by

$$
W_{2} \bar{x}=\rho g \int_{0}^{\theta} \pi\left(b^{2}-a^{2}\right) R^{2}\left(1-\cos \theta^{\prime}\right) d \theta^{\prime}=\rho g \pi\left(b^{2}-a^{2}\right) R^{2}(\theta-\sin \theta),
$$


where $\rho$ is the density of the preform material and $g$ is gravitational acceleration. Thus, using (1),

$$
\rho g \pi\left(b^{2}-a^{2}\right) R^{2}(\theta-\sin \theta)=\frac{k\left(b^{4}-a^{4}\right) \pi}{4},
$$

and hence

$$
k=(\theta-\sin \theta) \frac{4 \rho g R^{2}}{b^{2}+a^{2}} .
$$

The distance from the die axis to the centre of mass of the whole preform of weight $W$ is

$$
d=\frac{W_{2} \bar{x}}{W}=\frac{\rho g \pi\left(b^{2}-a^{2}\right) R^{2}(\theta-\sin \theta)}{W}=\frac{R^{2}(\theta-\sin \theta)}{L_{1}+R \theta},
$$

where $L_{1}$ is the length of the straight section of preform, above the bend.

\section{Analysis of bending}

Experimental results have been provided by the CEP-UA for solid glass preforms $(a=0)$ having radius $b=0.5 \mathrm{~cm}$ and total length $L=L_{1}+L_{2}=20 \mathrm{~cm}$. Bent ends have length $\mathrm{L}_{2}=\mathrm{R} \sin \theta \approx 4 \mathrm{~cm}$ and deviations $\delta=\mathrm{R}(1-\cos \theta) \approx$ $0.4 \mathrm{~cm}$, from which

$$
\frac{\mathrm{L}_{2}}{\delta}=\frac{\sin \theta}{1-\cos \theta}=\mathrm{f}(\theta)=10 .
$$

By graphing $f(\theta)$, or otherwise, we find $\theta \approx 0.2$ radians $\approx 12^{\circ}$. Then $R=$ $\mathrm{L}_{2} / \sin \theta \approx 20 \mathrm{~cm}$. For glass of density $\rho=2.5 \mathrm{~g} / \mathrm{cm}^{3},(2)$ and (3) yield

$$
\mathrm{k}=2.1 \mathrm{kPa} / \mathrm{cm} \text { and } \mathrm{d}=0.027 \mathrm{~cm} \text {, }
$$

while the average stress is

$$
\sigma_{0}=W / A=\rho g\left(L_{1}+R \theta\right)=4.9 \mathrm{kPa} .
$$


Thus, this analysis yields a linear stress profile across the $1 \mathrm{~cm}$ diameter of the die outlet with $3.8 \leqslant \sigma \leqslant 5.9 \mathrm{kPa}$. Given that, for extrusion of glass preforms, the applied pressure is $10-20 \mathrm{MPa}$, an asymmetry of order $\pm 10^{-3} \mathrm{MPa}$ is small and quite conceivable.

Next we consider a tube preform of the same external radius $b=0.5 \mathrm{~cm}$; thus $0<a<b$. If $\theta$ and $R$ are as above, that is, the bend geometry is similar to that for the rod preform, then equation (2) shows that $k$ is smaller. Thus, for a given bend geometry, less asymmetry in the stress profile is required for a tube preform than a rod preform. However, because the applied pressures used for extrusion of tube preforms are similar to those used for rod preforms, we expect a similar value of $k$. Then, if the bend radius $R$ is as for the rod preform, the bend angle $\theta$ must be larger. Unfortunately, we do not have experimental data to directly confirm this. That available to us is for tube preforms with external radius $b=0.5 \mathrm{~cm}$ and small internal radius $a=0.1 \mathrm{~cm}$. This internal radius is not sufficiently large to have a significant difference on the bend geometry which is very similar to that seen in the rod preforms.

A typical polymer has a density of $1.15 \mathrm{~g} / \mathrm{cm}^{3}$. Then, for a polymer preform, the stresses at the die outlet are $1.15 / 2.5=0.46$ of those that would pertain if the preform were of glass. If polymer preforms are extruded using pressures of the same magnitude as used for glass (10-20 MPa) then we expect the stress asymmetry at the die outlet, that is $k$, to be of a similar magnitude also. Then, from equation (2) we have

$$
\frac{\left(\theta_{p}-\sin \theta_{p}\right) R_{p}^{2}}{\left(\theta_{g}-\sin \theta_{g}\right) R_{g}^{2}}=\frac{\rho_{g}}{\rho_{p}}=\frac{2.5}{1.15}=2.17,
$$

where $\theta_{p}, R_{p}$ and $\rho_{p}$ are the bend angle and radius for a polymer preform and the polymer density, respectively, and $\theta_{g}, R_{g}$ and $\rho_{g}$ are those quantities for a similar glass preform. From this we expect more significant bending in polymer preforms than glass preforms, and this is indeed seen in practice. To keep the extent of bending in polymer preforms commensurate with that of glass preforms it is necessary to reduce the extrusion pressure to half or 
less of that used for glass. Thus, for polymer preforms extrusion pressures of $1-10 \mathrm{MPa}$ are used [3].

\section{Conclusions}

Bending during extrusion is often attributed to non uniformity in temperature and/or asymmetry in die geometry. These factors can be causes of bending in extrusion of optical fibre preforms. However, significant bending occurs when extruding plasticine (with no heating) through an axisymmetric rod die, using the extruder at the CEP-UA. The analysis conducted herein shows that preform bending can result from small asymmetries in the applied pressure, something which is seen as a distinct possibility with the experimental extruder in question.

For rod preforms made of glass of diameter $1 \mathrm{~cm}$, a linear stress profile across the die outlet diameter with a pressure difference of about $2 \mathrm{kPa}$ from one side to the other is sufficient to cause the bends seen in practice. This compares with applied pressures of around $20 \mathrm{MPa}$ used for extrusion of these preforms. Assuming a similar asymmetry of $\pm 1 \mathrm{kPa} / \mathrm{cm}$ in the pressure applied during extrusion, we have that a very small asymmetry in the applied pressure can result in significant bending of the preform.

Given the small magnitude of this asymmetry it seems, at first, that little can be done to control bending of preforms. However, this work also shows that applying a small force, of the same magnitude as the weight of the bent portion of a preform $\left(W_{2}=\rho g \pi\left(b^{2}-a^{2}\right) R \theta\right)$, to the end of the preform as it emerges from the die will prevent bending due to asymmetry in the applied pressure. For a rod preform of $1 \mathrm{~cm}$ diameter made from glass, this is a weight of about $0.08 \mathrm{~N}$ corresponding to a mass of 8 grams. Following this work, the extruder at the CEP-UA was modified to allow application of such a force as the preform emerges from the die. This has greatly alleviated the problem of preform bending and also provides more control in the event 
of non-isothermal conditions and asymmetries in die design [5].

Acknowledgements The author is grateful for the discussions with Assoc. Prof. David Clements on this problem, which led to the quasi-static model used here, and for the information supplied by Dr Heike EbendorffHeidepriem of the CEP-UA.

\section{References}

[1] G. Barakos and E. Mitsoulis. Non-isothermal viscoelastic simulations of extrusion through dies and prediction of the bending phenomenon, J. Non-Newtonian Fluid Mech., 62, 1996, 55-79. doi:10.1016/0377-0257(95)01385-7 C126

[2] H. Ebendorff-Heidepriem and T. M. Monro. Extrusion of complex preforms for microstructred optical fibres, Optics Express, 15, 2007, 15086-15092. doi:10.1364/OE.15.015086 C125

[3] H. Ebendorff-Heidepriem, Centre of Excellence in Photonics, The University of Adelaide. Personal communication, 2006-9. C127, C134

[4] A. Karagiannis, A. N. Hrymak and J. Vlachopoulos. Three-dimensional non-isothermal extrusion flows, Rheol. Acta, 28, 1989, 121-133. doi:10.1007/BF01356973 C126

[5] S. C. Warren-Smith, H. Ebendorff-Heidepriem, T. C. Foo, R. Moore, C. Davis and T.M. Monro. Exposed-core microstructured optical fibers for real-time fluorescence sensing, Optics Express, 17, 2009, 18533-18542. doi:10.1364/OE.17.018533 C126, C135 


\section{Author address}

1. Y. M. Stokes, School of Mathematical Sciences, The University of Adelaide, SA, Australia.

mailto:Yvonne.Stokes@adelaide.edu.au 